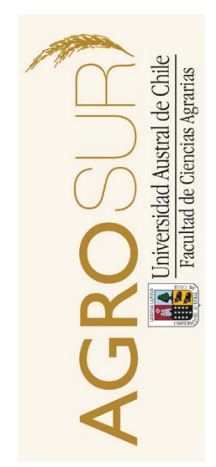

\title{
Dinámica vegetacional de praderas sembradas con especies nativas y naturalizadas con y sin aplicación de fertilizante
}

\section{Vegetational dynamics of sown pastures with native and naturalized species with and without fertilizer application}

\author{
Ramírez, M. ${ }^{a}$, Keim, J.P. ${ }^{b^{*}}$, López, I.F. ${ }^{b}$, Balocchi, 0. $^{b}$ \\ ${ }^{a}$ Escuela de Agronomía, Facultad de Ciencias Agrarias, Universidad Austral de Chile \\ ${ }^{b}$ Instituto de Producción Animal, Facultad de Ciencias Agrarias, Universidad Austral de Chile. Casilla 567, Valdivia, Chile.
}

ARTICLE INFO

Article history:

Received 11.06.14

Accepted 09.08.14

Keywords:

Bromus spp.

Botanical composition

Fertilization response

Original Research Article

Animal Science

${ }^{*}$ Corresponding author:

Juan Pablo Keim

E-mail address:

juan.keim@uach.cl
A B S T R A C T

A 6 year study was carried out at Santa Rosa research station, of the University Austral of Chile to determine the effect of type of pasture and fertilizer application on changes in the botanical composition and pasture and species production.

The evaluated pastures were: multi-species (PP), sown with Agrostis capillaris L., Arrhenatherum elatius ssp. bulbosus (Willd.) Schübler \& Martens, Bromus valdivianus Phil., Holcus lanatus L. y Lotus uliginosus Schkuhr; pasture sown with genetically improved species (PM) (Lolium perenne L. cv. Yatsyn y Trifolium repens L. cv. Huia); and a mixed pasture (PM+B) sown with Lolium perenne cv. Yatsyn, Trifolium repens cv. Huia and Bromus valdivianus.

The experimental design comprised a complete randomized blocks design ( 3 blocks), with factorial arrangement of the treatments (3 types of pasture $\mathrm{x} 2$ levels of fertilization) and the effect of year of evaluation as a repeated measure. Analysis of variance was performed using the MIXED procedure of SAS. The adjusted-Tukey with the PDIFF command was performed for the mean comparison. The multi-species pasture showed similar herbage mass accumulation to pastures sown with genetically improved species, across the six years of evaluation, indicating that there was not a specific pasture with a higher persistency. Fertilization increased herbage mass accumulation regardless the type of pasture. B. valdivianus and L. perenne increased their herbage mass accumulation and contribution with fertilizer application. However, B. valdivianus production was more stable over time, whereas the contribution of L. perenne tended to decline. Agrostis capillaris increased its contribution in non-fertilized pastures.

\section{RESUMEN}

Durante seis años se evaluó la dinámica vegetacional de praderas sembradas con especies nativas y naturalizadas sometidas a dos niveles de fertilización y su efecto en la producción de materia seca anual.

Las praderas fueron: pradera polifítica sembrada con Agrostis capillaris L., Arrhenatherum elatius ssp. bulbosus (Willd.) Schübler \& Martens, Bromus valdivianus Phil., Holcus lanatus L. y Lotus uliginosus Schkuhr (PP); pradera sembrada con con Lolium perenne L. cv. Yatsyn y Trifolium repens L. cv. Huia (PM); y pradera sembradacon Lolium perenne cv. Yatsyn, Trifolium repens cv. Huia y Bromus valdivianus (PM+B). El estudio se ajustó a un diseño experimental de bloques completos al azar (3 bloques), con arreglo factorial de los tratamientos (3 tipos de pradera x 2 niveles de fertilización), considerando el año de evaluación como medida repetida en el tiempo. Se realizó análisis de varianza y comparación de medias con el test de Tukey sobre las medias ajustadas. Praderas sembradas con especies nativas y naturalizadas (PP y PM+B) no difieren en producción de MS con praderas sembradas sólo con especies mejoradas (PM), sin presentar una mayor estabilidad en la producción de MS a lo largo del tiempo. Los tres tipos de pradera fertilizadas aumentaron sus rendimientos respecto a praderas sin fertilización. Tanto B. valdivianus como L. perenne se vieron favorecidos por los niveles de fertilidad, mientras que en el tiempo B. valdivianus se mantuvo más estable que L. perenne, la cual fue disminuyendo su presencia en todas las praderas. En las praderas sin fertilización se observó un aumento en la contribución de A. capillaris.

Palabras clave: Bromus spp., composición botánica, respuesta a la fertilización

\section{INTRODUCCIÓN}

La superficie de praderas en las décima y decimocuarta regiones se estima en 1.177.294,5 ha, de las cuales el 43,8\% corresponde a praderas naturalizadas, mientras que las artificiales y las mejoradas represen$\tan$ el 8,5 y $47,7 \%$ de la superficie, respectivamente (INE, 2007). 
Las praderas naturalizadas presentan baja productividad (menor a 4 ton MS ha-1 año-1) y marcada estacionalidad de la producción (más de $50 \%$ de la producción de MS durante la primavera), alcanzando distintos grados de degradación (Montaldo, 1973). Es una comunidad polifítica dominada fundamentalmente por gramíneas (Agrostis capillaris L., Holcus lanatus L., Lolium perenne L., Dactylis glomerata L., Bromus valdivianus Phil., Arrhenatherum elatius spp bulbosum L., Paspalum dasypleurum Kunze, Anthoxanthum odoratum L. y Lolium multiflorum Lam), con una proporción variable de especies de hoja ancha y una contribución de leguminosas que representa en general menos del $5 \%$ del rendimiento total anual de la pradera (Balocchi y López, 1996).

Existen tres estrategias para revertir estados severos de degradación en praderas: establecer una nueva pastura mediante siembra tradicional; recuperarlas por medio de una regeneración; y combinar prácticas de manejo como la sectorificación del predio, la regulación de la carga animal, el control del pastoreo, el control de plagas, el drenaje en suelos con problemas y la fertilización (Hampton et al., 1999). Incorporar especies mejoradas genéticamente como Lolium perenne L. y Trifolium repens L. a través de la siembra, aumenta la producción de MS, el valor nutritivo de las praderas, la resistencia al ataque de insectos y enfermedades, permite nivelar el suelo, incorporar sistemas de drenaje y mejorar la fertilidad del suelo (Kemp et al., 1999).

La composición botánica de las praderas establecidas o regeneradas se modifica como consecuencia de la incorporación de nuevas especies. Sin embargo, si las condiciones no favorecen el desarrollo de las especies sembradas, la pradera volverá a estar dominada por las especies originales (Wedderburn et al., 1996).

Existen antecedentes que especies forrajeras nativas y naturalizadas, pueden alcanzar producciones de MS similares a especies cultivadas cuando son sometidas a condiciones de manejo similar (Balocchi y López, 2001 y Jones, 1996) y favorecen una pradera más estable y con mayor persistencia en el tiempo, ya que se encuentran adaptadas a las condiciones características del ecosistema (Balocchi y López, 1996). Bromus unioloides L., Dactylis glomerata, Festuca arundinacea, Holcus lanatus, Lolium spp., Phleum pratense y Trifolium repens son especies que responden positivamente a la fertilización (Bailey, 2000 y Poozesh, 2010), por lo que deberían incrementar su participación en praderas fertilizadas (Cuevas, 1980).

La adición de fertilizante es una práctica que genera gran impacto en las praderas, al mejorar la producción de materia seca (Arzadun y Mestelan, 2009) y la calidad nutritiva del forraje (Poozesh et al., 2010). La fertilización y corrección de la acidez incrementan la producción de MS de las praderas, cuya magnitud depende de la condición inicial de ésta. Aumentos productivos como respuesta a la aplicación de cal, ocurren bajo condiciones de alta acidez (Besga et al., 1997), pudiendo no existir efecto en el corto plazo sobre la producción de MS, composición botánica y calidad nutritiva con niveles de pH superiores a 5,6 (Mijangos et al., 2010). La respuesta productiva a la fertilización es variable y depende la condición de la pradera (Brum et al., 2009). La respuesta productiva a la fertilización con macro nutrientes como nitrógeno, fósforo y potasio es mayor que al encalado, existiendo un efecto sinérgico entre ambas prácticas de manejo (Besga et al., 1997 y Poozesh et al., 2010). Poozesh et al. (2010), determinaron un aumento de 4,5 veces en la producción de MS producto de la fertilización y el encalado en una pradera sembrada con Dactylis glomerata. La fertilización y el encalado disminuyeron la contribución de Agrostis capillaris y Holcus lanatus, mientras que Lolium perenne y Trifolium repens no tuvieron respuesta. Sin embargo, el efecto de la fertilización sobre la composición botánica no es consistente. Brum (2009) y Butkuviene y Butkute (2008), observaron un aumento de gramíneas de alto valor forrajero producto de la fertilización, situación que no ocurrió en los trabajos de Pinto et al. (1995) y Poozesh et al. (2010). La corrección de acidez mediante enmiendas calcáreas no incrementó la presencia de especies de alto valor forrajero en los estudios de Mijangos et al. (2010), de Pinto et al. (1995) y de Poozesh et al. (2010).

La siembra de praderas permite incorporar rápidamente nuevas especies en praderas dominadas por especies de bajo potencial productivo. Sin embargo, las especies sembradas tienden a desaparecer y a ser reemplazadas por especies naturalizadas (Siebald et al., 1983). Así es como en cuatro temporadas de evaluación la contribución de las especies sembradas fluctuó entre un 63 y 68\%, lo que refleja la aparición de otras especies (Tharmaraj et al., 2008). Por ejemplo, cuando una pradera dominada por L. perenne deja de ser fertilizada, esta disminuye su contribución en desmedro de las especies de hoja ancha (Hoffman y Isselstein, 2005). En este sentido, incorporar especies nativas y naturalizadas como Dactylis glomerata, Festuca Arundinacea y Lolium multiflorum permitirían incrementar y mejorar la distribución de la producción anual (Tharmaraj et al., 2010) y la persistencia (Balocchi y López, 1996) en relación a praderas sembradas con L. perenne y T. repens.

El presente trabajo plantea como hipótesis que la incorporación de nutrientes al suelo estimula a praderas sembradas con especies nativas y naturalizadas, alcanzando rendimientos de materia seca similares a praderas establecidas en base a especies genéticamente mejoradas, y a su vez son más estables en el tiempo. El objetivo de este estudio fue evaluar la dinámica vegetacional de praderas establecidas con especies nativas, naturalizadas y genéticamente mejoradas con o sin adición de fertilizante, durante un período de 6 años de evaluación. 


\section{MATERIAL Y MÉTODO}

El estudio se realizó en la Estación Experimental Agropecuaria Austral, de la Universidad Austral de Chile (paralelo $39^{\circ} 47^{\prime} 26^{\prime \prime}$ latitud sur y meridiano $73^{\circ} 14^{\prime}$ $12^{\prime \prime}$ longitud oeste).

La información climática se obtuvo de la estación meteorológica de la Universidad Austral de Chile, ubicada en el Campus Isla Teja en Valdivia. La Figura 1 muestra los datos de temperaturas medias y precipitaciones a lo largo del año entre el período marzo de 2003 a marzo de 2009.

El ensayo consistió en la siembra de tres tipos de praderas, establecidas entre el 15 y 27 de septiembre del año 2002: pradera polifítica sembrada (PP) con 5 $\mathrm{kg} \mathrm{ha}^{-1} \mathrm{de}$ A. capillaris, $30 \mathrm{~kg} \mathrm{ha}^{-1}$ de A. elatius ssp. bulbosum, $15 \mathrm{~kg} \mathrm{ha}^{-1}$ de $B$. valdivianus, $8 \mathrm{~kg} \mathrm{ha}^{-1}$ de $H$. lanatus y $2 \mathrm{~kg} \mathrm{ha}^{-1}$ de L. uliginosus; pradera mixta sembrada con $30 \mathrm{~kg} \mathrm{ha}^{-1}$ de L. perenne cv. Yatsyn y $5 \mathrm{~kg} \mathrm{ha}^{-1} \mathrm{de} T$. repens cv. Huia (PM); y pradera sembrada con $15 \mathrm{~kg} \mathrm{ha}^{-1}$ de L. perenne cv. Yatsyn, $5 \mathrm{~kg} \mathrm{ha}^{-1} \mathrm{de} T$. repens cv. Huia y $30 \mathrm{~kg} \mathrm{ha}^{-1}$ de $B$. valdivianus (PM+B), culminando el estudio en mayo de 2009. Los tres tipos de praderas fueron establecidas y mantenidas con y sin aplicación de fertilizante bajo condiciones de secano, por lo cual el ensayo constó de seis tratamientos.
Previo al establecimiento, se eliminó la vegetación existente aplicando glifosato (2025 g ingrediente activo $\mathrm{ha}^{-1}$ ). Posteriormente se procedió a arar y rastrear el suelo para preparar una cama de semillas firme y homogénea. Estas labores se realizaron al final del periodo invernal de 2002. La siembra de las praderas se realizó en línea, con una sembradora manual Planet Junior.

El estudio consideró un período de seis años de evaluación, desde marzo de 2003 a marzo 2009. Los seis tratamientos, se distribuyeron aleatoriamente en tres bloques y cada parcela tuvo una dimensión de 7,6 x 3,3 m.

\section{Fertilización y criterios de cortes}

Las dosis de fertilizantes y enmienda calcárea utilizadas en el ensayo se determinaron tomando un análisis de suelo correspondiente a cada año de evaluación, siendo calculadas de acuerdo a la metodología propuesta por Pinochet (1990), considerando un rendimiento potencial de 14 ton MS ha ${ }^{-1}$ año $^{-1}$. Previo al establecimiento del ensayo se realizó un análisis de suelo a $20 \mathrm{~cm}$ de profundidad $\left(\mathrm{pH} \mathrm{CaCl}_{2}: 4,8\right.$; P-Olsen: 20,7 $\mathrm{mg} \mathrm{kg}^{-1}$; K-intercambiable: $128 \mathrm{mg} \mathrm{kg}^{-1}$; Suma de bases intercambiables: $1,95 \mathrm{cmol}+\mathrm{kg}^{-1}$; Al intercambiable: $0,3 \mathrm{cmol}+\mathrm{kg}^{-1}$; Saturación de Aluminio: 13,3\%). La dosis anual de nitrógeno se aplicó parcializada después

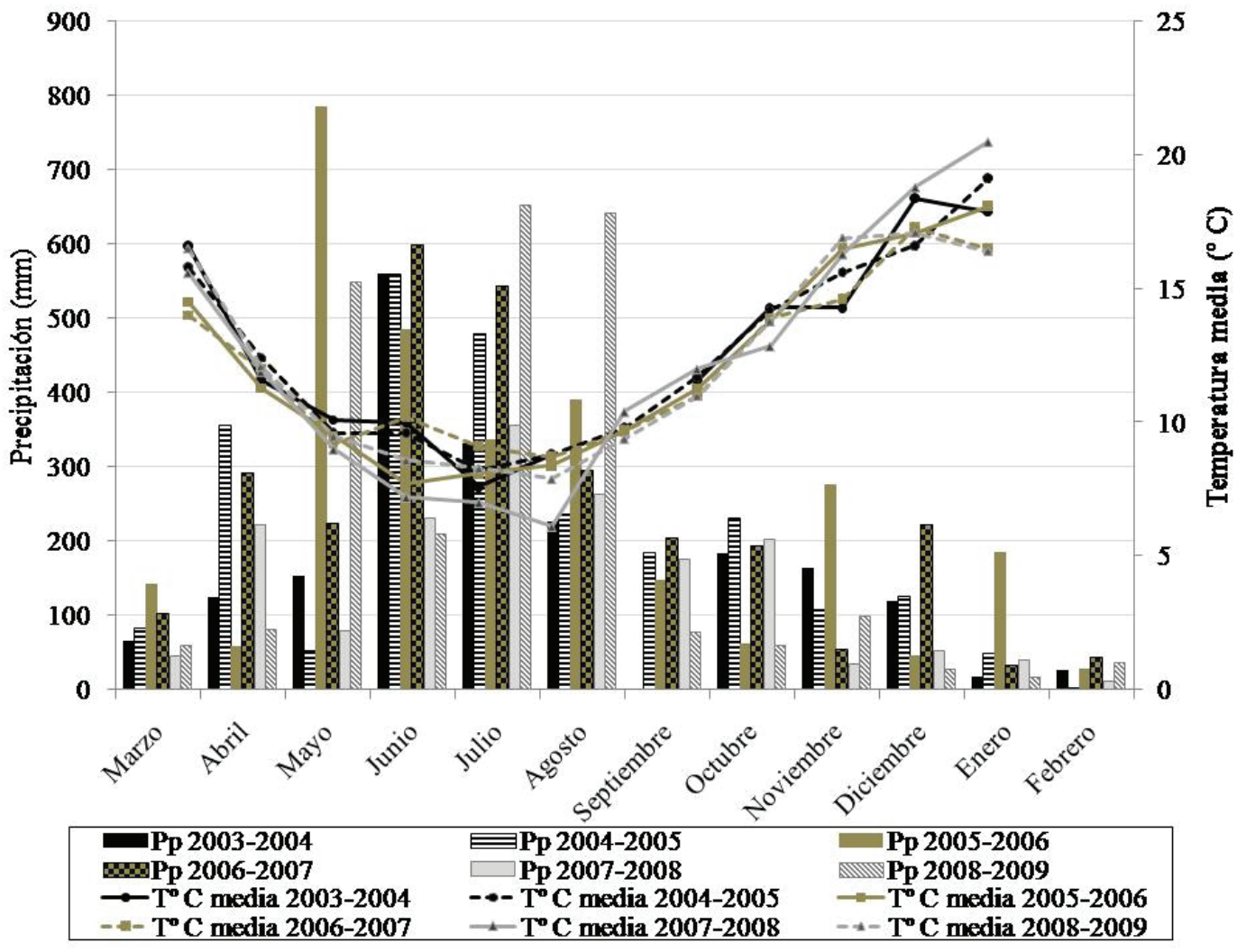

Figura 1. Condiciones meteorológicas durante el período experimental (marzo de 2003 a marzo de 2009) en Valdivia.

Figure 1. Meteorological conditions during the experimental period (March 2003 to March 2009) in Valdivia. 
de cada corte. Mientras que para las dosis de fósforo, potasio, magnesio se aplicaron en una sola dosis y en cobertera. La enmienda calcárea se aplicó en Mayo de cada año, en cobertera mediante encaladora. En el Cuadro 1, se detalla el rango de las dosis de fertilización y enmienda calcáreas del suelo, aplicadas durante los seis años de evaluación.

Como criterios de corte se procedió a realizar corte o muestreo cuando alguna de las praderas alcanzó una altura sin disturbar de $20 \mathrm{~cm}$, o en su defecto, 60 días después del último corte en caso de que la altura no se lograra. Por lo general, se realizaron ocho cortes para cada año de evaluación.

\section{Variables evaluadas}

Cada corte se realizó con una segadora de barra autopropulsada (Bertolini 140 L, Reggio Emilia, Italy) obteniendo una franja longitudinal de dimensiones conocidas $(6,5 \mathrm{~m} \times 1,1 \mathrm{~m})$, la cual fue pesada en verde y luego a partir de una submuestra se determinó el contenido de materia seca, obteniendo así el rendimiento de materia seca por corte (kg MS ha-1). La producción de MS anual correspondió a las sumatoria de las producciones de materia seca de los cortes en el período de un año.

La composición botánica se determinó para cada corte, para lo cual se extrajo una sub muestra de 200 g aproximadamente, de la cual se separaron manualmente las especies presentes en la muestra. Cada especie fue secada por separado en un horno de aire forzado a $60{ }^{\circ} \mathrm{C}$ por un período de 48 horas. La proporción de cada especie se calculó a partir de la relación entre el peso seco de la especie y el peso seco total de la submuestra. La producción de cada especie por corte se obtuvo a partir de la producción de MS anual y el porcentaje de cada especie en la pradera. La producción de MS anual de cada especie se determinó a partir de la sumatoria de la producción por corte de cada especie. Para el análisis de datos se consideró la producción de L. perenne, $B$. valdivianus y A capillaris, mientras que se agrupó a las otras especies de gramíneas (OG: A. elatius ssp. bulbosum, $A$. odoratum, D. glomerata, $H$. lanatus, $L$. multiflorum, Poa pratensis y Poa annua), leguminosas (LEG: L. uliginosus, T. repens y T. pratense) y hojas anchas (HA: Spegula arvensis, Rumex acetosella, Plantago lanceolata, Hypocaeris radicata, Leontodon nudicaulis, Cerastium arvensis, Dichondra repens, Taraxacum officinale y Ranunculus repens) producto de la baja presencia en los tratamientos.

\section{Diseño experimental y análisis estadístico}

El estudio se ajustó a un diseño de bloques completos al azar con arreglo factorial de los tratamientos (3 tipos de pradera x 2 niveles de fertilización) con tres repeticiones. El año de evaluación se incorporó al modelo como medida repetida en el tiempo, utilizando modelos mixtos (SAS, 2006).

Se consideró nivel de significancia de $\alpha=0,05$. Cuando existieron diferencias significativas, se realizó comparación de medias con el método de Tukey ajustado con el comando PDIFF de SAS.

\section{RESULTADOS Y DISCUSIÓN}

En el Cuadro 2 se presentan los niveles de significancia para el efecto del tipo de pradera, nivel de fertilización, año de evaluación y sus interacciones sobre todas las variables evaluadas. No se observó un efecto del tipo de pradera sobre la producción de MS anual, sin embargo, sí se observaron diferencias en la producción y contribución de las especies. La fertilización presentó un efecto significativo $(\mathrm{P}<0,05)$ sobre la producción de MS anual y las diferentes especies evaluadas, destacando que la incorporación de nutrientes favoreció el rendimiento de BV, LP, HA y OG (Cuadro 3). En tanto no se observó una interacción tipo de pradera*fertilización*año para ninguna de las variables evaluadas.

\section{Producción total y por especies}

La producción de MS anual fue similar entre los distintos tipos de pradera $(\mathrm{P}>0,05)$ con rendimientos de 6472, 6607 y $7150 \mathrm{~kg} \mathrm{MS} \mathrm{ha}^{-1} \mathrm{año}^{-1}$ para PM, PP y

Cuadro 1. Dosis de fertilización y enmienda calcárea aplicadas durante el período de evaluación.

Table 1. Levels of fertilizer and liming application throughout the experiment.

\begin{tabular}{|c|c|c|c|c|c|c|}
\hline \multirow[t]{2}{*}{ Elemento } & \multicolumn{6}{|c|}{ Dosis Anual (kg ha-1) } \\
\hline & $\mathrm{A} 1$ & $\mathrm{~A} 2$ & A3 & $\mathrm{A} 4$ & A5 & A6 \\
\hline Nitrógeno $(\mathrm{N})$ & 180 & 180 & 180 & 180 & 180 & 180 \\
\hline Fósforo $\left(\mathrm{P}_{2} \mathrm{O}_{5}\right)$ & 210 & 240 & 100 & 100 & 100 & 100 \\
\hline Potasio $\left(\mathrm{K}_{2} \mathrm{O}\right)$ & 264 & 47 & 168 & 224 & 60 & 185 \\
\hline Magnesio (MgO) & 210 & 75 & - & - & 10 & 46 \\
\hline Carbonato de Calcio $\left(\mathrm{CaCO}_{3}\right)$ & 1800 & 490 & - & 1800 & 1000 & 1800 \\
\hline
\end{tabular}


$\mathrm{PM}+\mathrm{B}$, respectivamente (Cuadro 3). Como era de esperar el tipo de pradera tuvo un efecto significativo sobre la producción de L. perenne, B. valdivianus, A. capillaris, OG y LEG $(\mathrm{P}<0,05)$. Para PM la mayor producción correspondió a L. perenne y HA. De igual modo en $\mathrm{PM}+\mathrm{B}$, $L$. perenne y $B$. valdivianus fueron las especies de mayor producción, mientras que en la $\mathrm{PP}, B$. valdivianus y HA tuvieron un mayor rendimiento.
En todas las praderas, el menor rendimiento fue obtenido por LEG y OG. Esto se puede deber, según Teuber y Elizalde (1999), a la presencia errática y poco definida de T. repens entre los diferentes tipos de pradera, debido a sus características de crecimiento rastrero de estolones, los cuales se ven fuertemente afectados por la falta de luz debido a la competencia de las otras especies presentes, principalmente las gramíneas por su hábito de creci-

Cuadro 2. Niveles de significancia para el efecto de tipo de pradera, nivel de fertilización, año de evaluación y sus interacciones.

Table 2. Level of significance for the effects of type of pasture, level of fertilization, year of evaluation and their interactions.

\begin{tabular}{|c|c|c|c|c|c|c|c|}
\hline & TP & $\mathrm{F}$ & A & $\mathrm{TP} * \mathrm{~F}$ & TP*A & $\mathrm{F}^{*} \mathrm{~A}$ & $\mathrm{TP} * \mathrm{~F}^{*} \mathrm{~A}$ \\
\hline \multicolumn{8}{|c|}{ Rendimiento (kg MS ha- ${ }^{-1}$ año ${ }^{-1}$ ) } \\
\hline PT & 0,423 & $<0,001$ & $<0,001$ & 0,750 & 0,975 & 0,015 & 0,997 \\
\hline $\mathrm{AC}$ & 0,044 & 0,001 & $<0,001$ & $<0,001$ & 0,030 & 0,005 & 0,104 \\
\hline BV & $<0,001$ & $<0,001$ & 0,005 & 0,539 & $<0,001$ & 0,001 & 0,989 \\
\hline LP & $<0,001$ & $<0,001$ & $<0,001$ & 0,044 & 0,025 & 0,357 & 0,996 \\
\hline GR & $<0,001$ & $<0,001$ & $<0,001$ & 0,0098 & 0,003 & 0,009 & 0,709 \\
\hline HA & 0,085 & 0,023 & $<0,001$ & 0,848 & 0,155 & 0,005 & 0,744 \\
\hline LEG & 0,025 & 0,022 & $<0,001$ & 0,768 & 0,415 & $<0,001$ & 0,178 \\
\hline \multicolumn{8}{|c|}{ Composición Botánica } \\
\hline$\% \mathrm{AC}$ & 0,212 & $<0,001$ & $<0,001$ & 0,018 & 0,530 & $<0,001$ & 0,751 \\
\hline$\% \mathrm{BV}$ & $<0,001$ & $<0,001$ & $<0,001$ & 0,252 & $<0,001$ & $<0,001$ & 0,459 \\
\hline$\% \mathrm{LP}$ & $<0,001$ & $<0,001$ & $<0,001$ & 0,053 & 0,012 & 0,391 & 0,936 \\
\hline$\% \mathrm{GR}$ & $<0,001$ & 0,312 & $<0,001$ & 0,514 & $<0,001$ & 0,005 & 0,979 \\
\hline$\% \mathrm{HA}$ & 0,029 & $<0,001$ & $<0,001$ & 0,717 & 0,473 & $<0,001$ & 0,985 \\
\hline \%LEG & 0,072 & 0,004 & 0,005 & 0,931 & 0,872 & 0,034 & 0,240 \\
\hline
\end{tabular}

TP: tipo de pradera; F: nivel de fertilización; A: año de evaluación; PT: Producción de MS anual; AC: Agrostis capillaris; HA: Hojas anchas; LP: Lolium perenne; BV: Bromus valdivianus; OG: Otras gramíneas; LEG: Leguminosas

Cuadro 3. Efecto del tipo de pradera sobre la producción de materia seca anual total y de las principales especies (kg MS ha-1 año-1) Table 3. Effect of type of pasture on total annual dry matter and main species yield (kg DM ha-1 year $\left.^{-1}\right)$.

\begin{tabular}{lrrrrr}
\hline & PM & PM+B & PP & EEM & Significancia \\
\hline PT & 6472 & 6608 & 7150 & 385 & 0,423 \\
AC & 936 & 804 & 994 & 88 & 0,044 \\
BV & 966 & 2074 & 3488 & 242 & $<0,001$ \\
LP & 2889 & 2231 & 481 & 279 & $<0,001$ \\
HA & 1469 & 1177 & 1172 & 107 & 0,085 \\
OG & 121 & 342 & 983 & 61 & $<0,001$ \\
LEG & 69 & 72 & 141 & 21 & 0,025 \\
\hline
\end{tabular}

PM: pradera sembrada con L. perenne cv. Yatsyn y T. repens cv. Huia; PM+B: pradera sembrada con L. perenne cv. Yatsyn, T. repens cv. Huia y B. valdivianus; PP: pradera sembrada con A. capillaris, A. elatius ssp. bulbosum, B. valdivianus, H. lanatus y L. uliginosus; PT: Producción de MS anual, HA: Hojas anchas, LP: Lolium perenne, BV: Bromus valdivianus, OG: Otras gramíneas, LEG: Leguminosas, AC: Agrostis capillaris, EEM: Error estándar de la media. 
miento erecto. Así mismo, el grupo OG al tener menores exigencias nutricionales en el suelo y al estar influenciadas con los tratamientos con fertilización tienen menor capacidad de competir con otras especies más agresivas y de rápido crecimiento (Balocchi y López, 1996).

No existió interacción entre tipo de praderas y nivel de fertilización sobre la producción total de materia seca anual $(\mathrm{P}>0,05)$, ya que los tres tipos de pradera sometidas a fertilización aumentaron sus rendimientos respecto a praderas sin fertilización (Cuadro 4). Concordando con los resultados obtenidos por Keim et al. (2014), quienes indican que al incorporar nutrientes al suelo como medida de mejoramiento de praderas naturales, se estimula a que aumenten la producción aproximadamente en un 50\%. Tal es el caso, que PM y PM+B incrementaron su producción en un $80 \%$, mientras que PP aumentó un 54\%, siendo las tres praderas fertilizadas las que alcanzaron mayor producción de MS anual, mientras que PM, PM+B y PP sin fertilización obtuvieron 4622,4699 y $5626 \mathrm{~kg} \mathrm{MS} \mathrm{ha}^{-1}$ año $^{-1}$ respectivamente, sin observarse diferencias significativas entre tipos de pradera.

Para la producción de MS anual de L. perenne, OG y A. capillaris se observó una interacción significativa entre tipo de pradera y nivel de fertilización $(\mathrm{P}<0,05)$. En el caso de L. perenne en PM y PM+B aumentó la producción de MS entre 228 y $215 \%$ al ser fertilizadas (respecto a PM y PM+B sin aplicación de fertilizante), mientras que para PP no hubo diferencias al fertilizar, ya que $L$. perenne no fue sembrada en esta pradera (Cuadro 4). En el caso de OG, PP con y sin fertilización alcanzaron mayor rendimiento, destacando que con fertilización aumentó un $70 \%$ en relación a PP sin fertilización. En el caso de la PM+B ésta aumentó respecto del tratamiento sin fertilización un $219 \%$. No obstante la producción en PM con fertilización fue similar a PM sin fertilización, dado que PP fue sembrada con distintas gramíneas (A. capillaris, A. elatius ssp. bulbosum, B. valdivianus, $H$. lanatus), mientras que la $\mathrm{PM}+\mathrm{B}$ fue sembrada con $L$. perenne, B. valdivianus y T. repens, y PM sólo con $L$. perenne y $T$. repens. Las especies gramíneas sembradas en PP serían capaces de desarrollar altas tasas de crecimiento, teniendo mayor ventaja por la diversidad de especies que le confiere un mayor rango para tolerar a los diferentes estrés climático-ambientales que una pradera basada en L. perenne y T. repens (Keim et al., 2014).

La producción de $A$. capillaris fue mayor en PM y $\mathrm{PM}+\mathrm{B}$ sin fertilización respecto a $\mathrm{PM}$ y $\mathrm{PM}+\mathrm{B}$ al ser fertilizadas, mientras que para PP no hubo diferencias producto de la aplicación de fertilizante. Por el contrario, Pinochet et al. (1999), observaron que en A. capillaris aumenta su producción cuando es sometida a fertilización en praderas naturalizadas y en monocultivo, respectivamente. Ésta interacción entre tipo de pradera y aplicación de fertilizante sugiere que A. capillaris aumenta su producción en praderas que no reciben fertilización cuando aparece espontáneamente, mientras que cuando es sembrada la producción de MS no se ve influenciada por la adición de fertilizante, comportándose como una especie tolerante de acuerdo a lo propuesto por Grime (1989).

En el caso de las especies de B. valdivianus, HA y LEG, no hubo interacción significativa $(\mathrm{P}>0,05)$. En $B$. valdivianus, la tendencia fue similar, ya que incrementó su producción con la fertilización en las tres praderas, alcanzando $4511 \mathrm{~kg} \mathrm{MS} \mathrm{ha}^{-1} \mathrm{año}^{-1}$ en PP. Esto concuer-

Cuadro 4. Interacción entre tipo de pradera y fertilización sobre la producción media de materia seca total y de las diferentes especies (kg MS ha-1 año-1).

Table 4. Interaction between type of pasture and level of fertilization on average total dry matter and species yield (kg DM $\mathrm{ha}^{-1} \mathrm{yr}^{-1}$ ).

\begin{tabular}{lrrrrrrrr}
\hline & & \multicolumn{1}{c}{ SF } & & & CF & & EEM & Significancia \\
\hline & PM & PM+B & \multicolumn{1}{c}{ PP } & PM & PM+B & PP & & \\
\hline PT & 4622 & 4699 & 5626 & 8322 & 8516 & 8674 & 545 & 0,750 \\
AC & 1120 & 1011 & 924 & 752 & 596 & 1064 & 103 & $<0,001$ \\
BV & 266 & 1034 & 2465 & 1667 & 3114 & 4511 & 343 & 0,539 \\
LP & 1758 & 1414 & 336 & 4019 & 3047 & 625 & 394 & 0,044 \\
OG & 129 & 215 & 727 & 114 & 470 & 1239 & 86 & 0,010 \\
HA & 1314 & 998 & 1077 & 1625 & 1355 & 1265 & 151 & 0,848 \\
LEG & 88 & 97 & 181 & 50 & 47 & 101 & 29 & 0,768 \\
\hline
\end{tabular}

SF: Sin aplicación de fertilizante; CF: con aplicación de fertilizante; PM: pradera sembrada con L. perenne cv. Yatsyn y T. repens cv. Huia; PM+B: pradera sembrada con L. perenne cv. Yatsyn, T. repens cv. Huia y B. valdivianus; PP: pradera sembrada con A. capillaris, A. elatius ssp. bulbosum, B. valdivianus, H. lanatus y L. uliginosus; PT: Producción total, HA: Hojas anchas, LP: Lolium perenne, BV: Bromus valdivianus, OG: Otras gramíneas, LEG: Leguminosas, AC: Agrostis capillaris, EEM: Error estándar de la media. 
da con López et al. (1997) y Balocchi y López (2001), quienes señalan que $B$. valdivianus es una especie que domina en sitios de alta fertilidad y alto potencial productivo.
Se observaron interacciones significativas entre aplicación de fertilizante y año de evaluación $(\mathrm{P}<0,05)$ para PT, B. valdiviaus, A. capillaris, HA, OG y LEG (Cuadro 5), las cuales pueden ser explicadas en parte por

Cuadro 5. Interacción entre aplicación de fertilizante y año de evaluación en la producción media total y de las especies. (kg MS ha' ${ }^{-1}$ año $0^{-1}$.

Table 5. Interaction between fertilizer application and year of evaluation on mean total and species yield. $\left(\mathrm{kg} \mathrm{DM} \mathrm{ha}^{-1} \mathrm{yr}^{-1}\right)$.

\begin{tabular}{|c|c|c|c|c|c|c|}
\hline & AÑO 1 & AÑO 2 & AÑO 3 & AÑO 4 & AÑO 5 & AÑO 6 \\
\hline \multicolumn{7}{|l|}{ PT } \\
\hline $\mathrm{CF}$ & 10350 & 8989 & 8254 & 10610 & 5993 & 7129 \\
\hline SF & 9015 & 6399 & 6371 & 4597 & 1774 & 1739 \\
\hline Significancia & \multicolumn{6}{|c|}{0,015} \\
\hline EEM & \multicolumn{6}{|c|}{770} \\
\hline \multicolumn{7}{|l|}{ HA } \\
\hline $\mathrm{CF}$ & 2054 & 1572 & 1172 & 1682 & 185 & 1830 \\
\hline SF & 1630 & 800 & 1184 & 2054 & 385 & 724 \\
\hline Significancia & \multicolumn{6}{|c|}{0,005} \\
\hline EEM & \multicolumn{6}{|c|}{213} \\
\hline \multicolumn{7}{|l|}{ LP } \\
\hline $\mathrm{CF}$ & 4096 & 3176 & 2876 & 3395 & 914 & 925 \\
\hline SF & 3321 & 1567 & 1352 & 492 & 192 & 94 \\
\hline Significancia & \multicolumn{6}{|c|}{0,357} \\
\hline EEM & \multicolumn{6}{|c|}{558} \\
\hline \multicolumn{7}{|l|}{ BV } \\
\hline CF & 3067 & 3213 & 2069 & 3161 & 3631 & 3446 \\
\hline SF & 3346 & 2292 & 869 & 408 & 299 & 314 \\
\hline Significancia & \multicolumn{6}{|c|}{0,001} \\
\hline EEM & \multicolumn{6}{|c|}{485} \\
\hline \multicolumn{7}{|l|}{ GR } \\
\hline $\mathrm{CF}$ & 526 & 218 & 720 & 1139 & 519 & 524 \\
\hline SF & 334 & 109 & 891 & 400 & 317 & 89 \\
\hline Significancia & \multicolumn{6}{|c|}{0,009} \\
\hline EEM & \multicolumn{6}{|c|}{122} \\
\hline \multicolumn{7}{|l|}{ LEG } \\
\hline $\mathrm{CF}$ & 186 & 163 & 18 & 20 & 5.6 & 2.6 \\
\hline SF & 72 & 156 & 350 & 104 & 42 & 8.1 \\
\hline Significancia & \multicolumn{6}{|c|}{$<0,001$} \\
\hline EEM & \multicolumn{6}{|c|}{41} \\
\hline \multicolumn{7}{|l|}{ AC } \\
\hline $\mathrm{CF}$ & 543 & 755 & 1468 & 992 & 554 & 512 \\
\hline SF & 445 & 1517 & 1746 & 1154 & 629 & 620 \\
\hline Significancia & \multicolumn{6}{|c|}{0,005} \\
\hline EEM & \multicolumn{6}{|c|}{128} \\
\hline
\end{tabular}

PT: Producción de MS anual, PM: Pradera sembrada, PM+B: Pradera mejorada, PP: Pradera polifítica, HA: Hojas anchas, LP: Lolium perenne, BV: Bromus valdivianus, GR: Otras gramíneas, LEG: Leguminosas, AC: Agrostis capillaris, CF: Con fertilización; SF: Sin Fertilización; EEM: Error estándar de la media. 
los cambios ambientales relacionados con temperatura y precipitaciones. En el caso de la producción de MS anual, la interacción se ve reflejada en que para el primer año ésta es similar entre praderas con y sin fertilización; mientras que a partir del segundo año las praderas fertilizadas tienen mayor producción; lo cual se puede explicar producto del aporte natural y la mineralización de nutrientes desde el suelo durante el primer año, y la reposición de los nutrientes extraídos mediante fertilización de mantención durante los demás años de estudio. Es así que Pinochet et al. (2000), indican que las praderas permanentes tienen un mayor potencial de mineralización de $\mathrm{N}$, el cual se produce por un mayor ingreso en cantidad y calidad de residuos.

Las especies de hojas anchas que tuvieron mayor producción respondieron a la fertilización en el segundo y sexto año, sin observarse respuestas para los demás años. Entre las más comunes, se encontraban Hypochaeris radicata, Rumex acetosella y Plantago lanceolata que aumentaban su participación en verano y primavera.

Para el caso de B. valdivianus y OG se observó que los tratamientos con fertilización alcanzaron mayores producciones a partir del segundo y cuarto año respectivamente. En el caso LEG ocurrió una mayor producción en tratamientos fertilizados durante el primer año, mientras que en el tercer y cuarto año la producción fue mayor en tratamientos sin fertilización. No obstante, la producción de LEG disminuyó en el tiempo alcanzando niveles inferiores a los $100 \mathrm{~kg} \mathrm{MS} \mathrm{ha-1}^{-1} \mathrm{ano}^{-1}$ en los últimos dos años. Según Balocchi (2007), la disminución del trébol no se debe a un efecto detrimental del fertilizante, sino a un incremento en la competencia ejercida por las especies gramíneas que dominan las praderas. En el caso de A. capillaris se observó una mayor producción en las praderas sin fertilización para los años 2 y 3 , sin observarse diferencias entre niveles de fertilización para los años 1, 4, 5 y 6.

\section{Composición botánica según tipo de pradera, fertilización y dinámica vegetacional.}

Se observaron diferencias significativas $(\mathrm{P}<0,05)$ entre tipos de pradera para la presencia de L. perenne, B. valdivianus, $\mathrm{HA}$ y $\mathrm{OG}$, mientras que A. capillaris y LEG fueron similares $(\mathrm{P}>0,05)$ (Figura 2). Lolium perenne representó en promedio un $34,8 \%$ en PM, mientras que en PM+B y PP se presentó en un 26,7\% y 6,1\%, respectivamente. Por otra parte, B. valdivianus alcanzó una mayor proporción en PP con 40,7\%, 30,5\% en PM+B y $15,7 \%$ en PM. Resultados similares para B. valdivianus fueron observados en praderas sembradas con especies naturalizadas en el sur de Chile (Pacheco, 1997; Jaramillo, 2003). Las especies de hoja ancha representaron un 25,7\% para PM, siendo este mayor que en PP y PM+B con 20,3 y $20,0 \%$, respectivamente; valores similares a los observados por Castro (1996), en praderas sembradas de L. perenne y T. repens $(21,9 \%)$ en el sur de Chile.

La presencia de OG fue baja en PM+B y PM con 5,6 y $2,7 \%$ respectivamente, teniendo un mayor aporte a la pradera polifítica con $14,8 \%$. No obstante, esto refleja la disminución de las especies sembradas A. elatius ssp. bulbosum y H. lanatus, producto de una menor habilidad competidora respecto a $B$. valdivianus. La ventaja de establecer praderas con un mayor número de especies, radicaría en que la diversidad de especies que confiere un mayor rango para tolerar los diferentes estreses climático-ambientales. (López et al. 2009), no obstante tanto en este estudio como en los de Deak et al. (2007) y Keim et al. (2014), se observó que praderas polifíticas sembradas terminan siendo dominadas por dos o tres especies, razón por la cual la elección de determinadas especies resulta más relevante que el número de especies presentes en una mezcla.

En el caso de LEG y A. capillaris, éstas no tuvieron diferencias significativas entre tipo de praderas $(\mathrm{P}>0,05)$ (Figura 2). A. capillaris contribuyó en alrededor de un $18 \%$ en la tres praderas, mientras que la presencia de LEG no superó el 3\% de la composición botánica, lo cual coincide con lo señalado por Balocchi y López (1996), quienes indican que las leguminosas tienen una contribución menor al 5\% del rendimiento total anual en pradera praderas del sur de Chile.

Respecto al efecto de la fertilización sobre la composición botánica de la pradera se observó un efecto significativo sobre la presencia de todas las especies excepto de OG. En el caso de L. perenne y B. valdivianus, éstas aumentaron su contribución de $17,4 \%$ a $27,6 \%$ y 17,7 a $37,8 \%$ respectivamente, producto de la fertilización (Figura 2). Balocchi (2007) señala que, en general, las especies gramíneas responden positivamente a la incorporación de nutrientes al suelo. Tanto B. valdivianus como L. perenne dominan en sitios de alta fertilidad y por lo general se encuentran compitiendo entre ellas por nichos ecológicos (Balocchi y López, 2001; López et al., 1997). De este modo, el crecimiento de éstas especies está limitado por la competencia que ejercen sobre ellas, respecto de la tasa de captura de nutrientes, del uso del espacio y el grado de accesibilidad a la luz (Tilman y Downing, 1994; Tow, 2001). En el caso de HA y A. capillaris, éstas disminuyeron su contribución en las praderas fertilizadas. Praderas con bajos niveles de nutrientes en el suelo, suelen ser dominadas por especies tolerantes a situaciones de estrés como son $A$. capillaris, $H$. radicata, L. nudicaulis; no obstante cuando las condiciones de estrés son corregidas, las especies tolerantes comienzan a ser desplazadas por especies competidoras (López et al., 1997; López y Valentine, 2003). Es así como Siebald et al. (2002), observaron que al cabo de cuatro años de fertilización A. capillaris disminuyó en un 22\%.

Se observó una interacción significativa $(\mathrm{P}<0,05)$ entre nivel de fertilización y los años de evaluación para 
a)
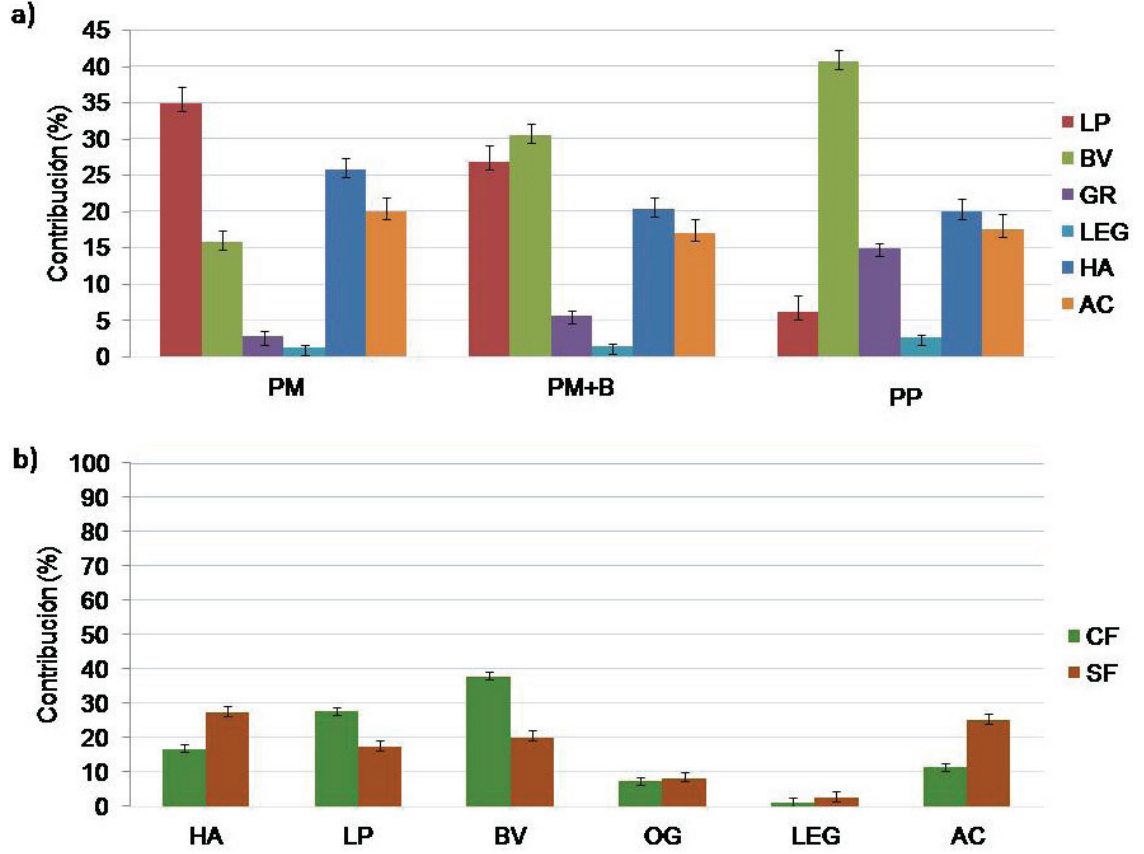

Figura 2. Contribución (\%) de las especies: (a) efecto pradera; (b) efecto fertilización (CF y SF). PM: pradera sembrada con L. perenne cv. Yatsyn y T. repens cv. Huia; PM+B: pradera sembrada con L. perenne cv. Yatsyn, T. repens $\mathrm{cv}$. Huia y B. valdivianus; PP: pradera sembrada con A. capillaris, A. elatius ssp. bulbosum, B. valdivianus, H. lanatus y L. uliginosus; HA: Hojas anchas; LP: Lolium perenne; BV: Bromus valdivianus; OG: Otras gramíneas; LEG: Leguminosas; AC: Agrostis capillaris; SF: Sin aplicación de fertilizante; CF: con aplicación de fertilizante. Barras de error indican error estándar de la media.

Figure 2. Contribution (\%) of species: (a) type of pasture effect; (b) fertilizer application effect (CF and SF). PM: sown pasture with L. perenne cv. Yatsyn and T. repens cv. Huia; PM+B: sown pasture with L. perenne cv. Yatsyn, T. repens cv. Huia and B. valdivianus; PP: sown pasture with A. capillaris, A. elatius ssp. bulbosum, B. valdivianus, H. lanatus and L. uliginosus; HA: Broad leaf species; LP: Lolium perenne; BV: Bromus valdivianus; OG: Other grasses; LEG: Legumes; AC: Agrostis capillaris; SF: without fertilizer application; CF: with fertilizer application. Error bars denote standard error of the mean.

A. capillaris, B. valdivianus, OG, HA y LEG (Figura 3). En el caso de $B$. valdivianus la interacción radica en que para los dos primeros años no se aprecian diferencias entre niveles de fertilización, no obstante, en los tratamientos con fertilización se observó un incremento en el tiempo desde $28,1 \%$ para el año 1 a $48,3 \%$ para el año 6 , siendo relevante el aumento ocurrido en los años 4 y 5 ; mientras que en praderas sin fertilización tuvo una tendencia decreciente, aportando 31,4\% el primer año y finalizando con 16,9\%. Esta interacción refleja la importancia de realizar estudios de largo plazo cuando se estudia la dinámica vegetacional, puesto que de lo contrario se pueden tomar conclusiones erróneas.

Para A. capillaris $(\mathrm{P}<0,01)$ las diferencias producto de la aplicación de fertilizante se reflejaron a partir del segundo año, alcanzando entre 25 y $30 \%$ al finalizar el estudio en las praderas sin fertilización, mientras que en praderas fertilizadas la contribución fue menor, variando entre 8 y 13,5\%. A. capillaris tiene mayor participación en praderas sin fertilización, dado que crece sobre suelos semi-húmedos a húmedos, pobres o poco fértiles y generalmente ácidos de pH 5-6 (Dietl et al.,
2009). López et al. (1997), indican que un aumento en los niveles de acidez y de aluminio propicia el ambiente para que especies como A. capillaris y A. odoratum ingresen, en desmedro de aquellas que no logran tolerar estos niveles de estrés como $B$. valdivianus y L. perenne.

En las especies de hojas anchas se observa una menor presencia producto de la fertilización, sin embargo, ésta aumentó en el sexto año, probablemente producto de otras situaciones de estrés ambiental como las bajas precipitaciones en verano (Figura 1), lo que puede haber afectado el crecimiento de especies gramíneas siendo desplazadas por HA; en tanto en el tratamiento sin fertilización la contribución aumentó a partir del tercer año, alcanzando un $42,7 \%$ al final del estudio. Cuevas (1980) y Grime et al. (1989), señalan que especies de hoja ancha como R. acetosella, P. lanceolata, L. nudicaulis, aumentan su participación en los tratamientos sin fertilización, debido a que tienen una mayor capacidad para competir en suelos de menor fertilidad.

Respecto a la interacción entre tipo de pradera y año de evaluación se observaron efectos significativos $(\mathrm{P}<0,05)$ sobre la contribución de B. valdivianus, L. pe- 

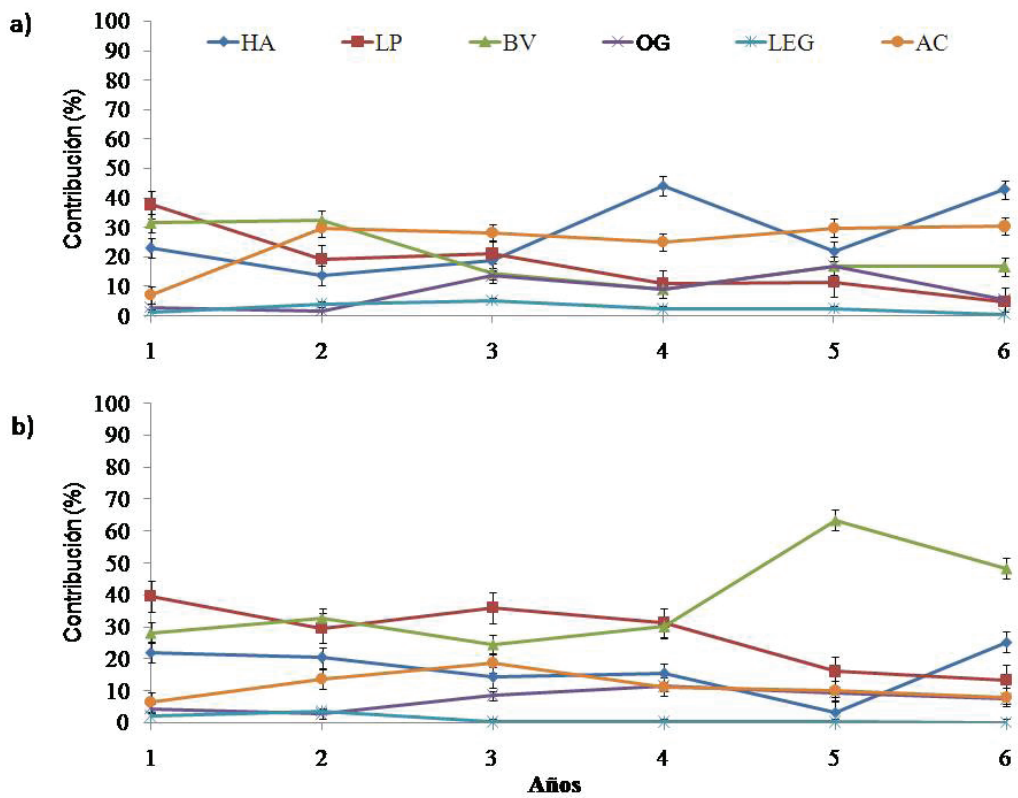

Figura 3. Contribución de las principales especies durante los 6 años de estudio (a) tratamiento sin fertilización, (b) con fertilización. HA: Hojas anchas, LP: Lolium perenne, BV: Bromus valdivianus, OG: Otras gramíneas, LEG: Leguminosas, AC: Agrostis capillaris. Barras de error indican error estándar de la media.

Figure 3. Contribution of the main species throughout the 6 years of experiment (a) without fertilizer application, (b) with fertilizer application. HA: Broad leaf species; LP: Lolium perenne; BV: Bromus valdivianus; OG: Other grasses; LEG: Legumes; AC: Agrostis capillaris; Error bars denote standard error of the mean.

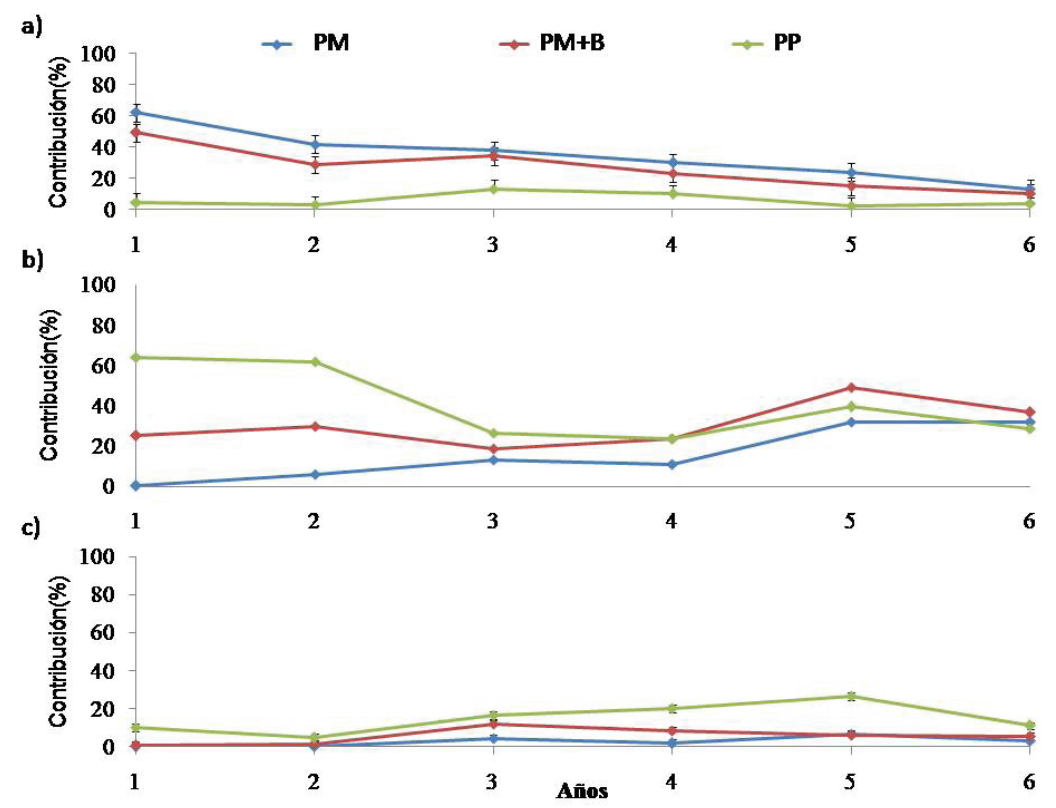

Figura 4. Interacción entre tipo de pradera y años de evaluación sobre la contibución de: (a) Lolium perenne (b) Bromus valdivianus y (c) Otras gramíneas. PM: pradera sembrada con L. perenne cv. Yatsyn y T. repens cv. Huia; PM+B: pradera sembrada con L. perenne cv. Yatsyn, T. repens cv. Huia y B. valdivianus; PP: pradera sembrada con A. capillaris, A. elatius ssp. bulbosum, B. valdivianus, H. lanatus y L. uliginosus; Barras de error indican error estándar de la media.

Figure 4. Interaction between type of pasture and years of evaluation on the contribution of (a) Lolium perenne (b) Bromus valdivianus and (c) Other grasses. PM: sown pasture with L. perenne cv. Yatsyn and T. repens cv. Huia; PM+B: sown pasture with L. perenne cv. Yatsyn, T. repens cv. Huia and B. valdivianus; PP: sown pasture with A. capillaris, A. elatius ssp. bulbosum, B. valdivianus, H. lanatus and L. uliginosus; Error bars denote standard error of the mean. 
renne y OG (Figura 4). La presencia de L. perenne fue disminuyendo en el tiempo en aquellas praderas donde fue sembrada (PM y PM+B), mientras que en PP su presencia se mantuvo relativamente baja. Estos resultados coinciden con Jones (1996), quien señala que $L$. perenne presenta baja persistencia en condiciones de secano, ya que no es capaz de tolerar el estrés hídrico.

En el caso de B. valdivianus se aprecia que un aumento sostenido en el tiempo en $\mathrm{PM}$ y $\mathrm{PM}+\mathrm{B}$, mientras que en PP disminuyó hacia el tercer año para luego estabilizarse y alcanzar entre un 30 y 40\% en todas las praderas.

Finalmente, la interacción para OG radica en un aumento en PP a partir del tercer año, para luego disminuir en el sexto año; mientras que en $\mathrm{PM}+\mathrm{B}$ disminuyó a partir del tercer año y en PM tuvo una baja presencia.

\section{CONCLUSIONES}

El presente estudio reveló que bajo las condiciones experimentales, la incorporación de especies nativas y naturalizadas como Agrostis capillaris, Arrhenatherum elatius ssp. bulbosum, Bromus valdivianus y Holcus lanatus en la siembra, permite alcanzar niveles de producción similares a praderas sembradas con especies como Lolium perenne y Trifolium repens, sin presentar una mayor estabilidad en la producción de MS a lo largo del tiempo. Por otro lado, la fertilización aumentó la producción anual consistentemente en todos los tipos de pradera a partir del segundo año. La dinámica vegetacional fue específica para cada tipo de pradera, existiendo variaciones según el nivel de fertilización. Tanto $B$. valdivianus como L. perenne se vieron favorecidos por la aplicación de fertilizante. Sin embargo, B. valdivianus se mantuvo más estable en el tiempo que L. perenne. Al contrario, la contribución de A. capillaris aumentó en las praderas que no recibieron fertilización.

\section{AGRADECIMIENTOS}

El presente estudio fue financiado por la Dirección de Investigación y Desarrollo de la Universidad Austral de Chile (Código Proyecto: DID 12S-2002-37). Los autores desean agradecer el trabajo realizado por Julio Vera, Pablo Fernández, Guisela Catalán, Jorge Gómez, Daniel Avendaño y Jaime Contreras. Los autores declaran que no existen conflictos de interés en el presente trabajo.

\section{REFERNECIAS}

ARZADUN, M.J., MESTELAN, S. A. 2009. Late Summer Management Can Improve Forage Yield Distribution and $\mathrm{Nu}-$ tritive Value in Temperate Grassland. Agronomy Journal 101: 584-591.

BAILEY, J.S. 2000. Influence of sward botanical composition on performance of cool temperate grassland under contrasting nitrogen fertilization regimes. Communications in Soil Science and Plant Analysis 31: 2855-2864.

BALOCCHI, O., LÓPEZ, I. 1996. Especies pratenses nativas y naturalizadas del sur de Chile. In: Latrille, L. (Ed.), Producción Animal. Universidad Austral de Chile, Facultad de Ciencias Agrarias, Instituto de Producción Animal. Serie B - 20. Valdivia, pp. 65-81.

BALOCCHI, O., LÓPEZ, I. 2001. Rol de las especies pratenses nativas y naturalizadas en las praderas permanentes del Sur de Chile. In: García Cretton, (Eds.), Proceedings. Simposio Internacional en Producción Animal y Medio Ambiente. Pontificia Universidad Católica de Chile, Santiago, Chile. pp. 285-299.

BALOCCHI, O. 2007. Mejoramiento de praderas naturales del sur de Chile. In: Hervé, M. (Ed.), Producción ovina: desde el suelo a la gestión. Fundación para la Innovación Agraria. Santiago, Chile. pp. 13-21.

BESGA, G., PINTO, M. RODRÍGUEZ, F.A., LÓPEZ, F.A., BALCAZAR, N. 1997. Agronomic and nutritional effects of LinzDonawitz slag application to two pastures in Northern Spain. Nutrient Cycling in Agroecosystems 46: 157-167.

BRUM, O.B., LOPEZ, S., GARCÍA, R., ANDRÉS, S., CALLEJA, A. 2009. Influence of harvest season, cutting frequency and nitrogen fertilization of mountain meadows on yield, floristic composition and protein content of herbage. Revista Brasileira De Zootecnia-Brazilian Journal of Animal Science 38: 596-604.

BUTKUVIENE, E. y BUTKUTE, R. 2008. Effects of pasture improvement measures on sward productivity, botanical and chemical composition. žemès ūkio mokslai 15: 46-52.

CASTRO, F. 1996. Evaluación del rendimiento y calidad nutritiva de una pradera naturalizada establecida bajo dos niveles de fertilización en el sur de Chile. Tesis Ing. Agr. Universidad Austral de Chile. 113 p.

CUEVAS, E. 1980. Manejo y utilización de praderas.. Universidad Austral de Chile, Facultad de Ciencias Agrarias, Instituto de Producción Animal. Serie b-2, Valdivia.

CHILE, INSTITUTO NACIONAL DE ESTADÍSTICAS (INE). 2007. VII Censo Agropecuario.

DEAK, A., HALL, M.H., SANDERSON, M.A., ARCHIBALD, D.D. 2007. Production and nutritive value of grazed simple and complex forage mixtures. Agronomy Journal 99: 814-821.

DIETL, W., FERNÁNDEZ, F. 2009. Manejo sostenible de praderas. Su flora y vegetación. Boletín INIA № 187, Instituto de Investigaciones Agropecuarias, Cauquenes, Chile.

GRIME, J., HODGSON, J., HUNT, R. 1989. Comparative plant ecology: A functional approach to common British species. Unit of comparative plant ecology (NERC), Department of plant science, University of Sheffield, England. 
HAMPTON, J., KEMP, P., WHITE, J. 1999. Pasture establishment. In: White, J., Hodgson, J. (Eds.), New Zealand Pasture and Crop Science. Oxford University press. pp. 101116.

HOFMANN, M., ISSELSTEIN, J. 2005. Species enrichment in an agriculturally improved grassland and its effects on botanical composition, yield and forage quality. Grass and Forage Science 60: 136-145.

JARAMILLO, C. 2003. Dinámica vegetacional y rendimiento de una pradera establecida en base a especies nativas y naturalizadas en su tercer año de producción.Tesis Lic. Agr. Universidad Austral de Chile, 126 p.

JONES, C.E. 1996. Pastoral value and production from native pastures. New Zealand Journal of Agricultural Research 39: 449-456.

KEIM, J., LOPEZ, I., BALOCCHI, O. 2014. Sward herbage accumulation and nutritive value as affected by pasture renovation strategy. Grass and Forage Science doi: 10.1111/gfs.12115.

KEMP, P., CONDRON, L., MATTHEW, C. 1999. Pasture species and cultivars. In: White, J., Hodgson, J. (Eds.), New Zealand Pasture and Crop Science. Oxford University press. pp. 83-100.

LÓPEZ, I., BALOCCHI, O., LAILHACAR, P., OYARZÚN, C. 1997. Caracterización de sitios de crecimiento de seis especies pratenses nativas y naturalizadas del dominio húmedo de Chile. Agro Sur 25: 62-80.

LÓPEZ, I., VALENTINE, I. 2003. Rol de la diversidad pratense y de los grupos funcionales de especies sobre la condición de la pradera y su estabilidad. Agro Sur 31: 60-76.

LÓPEZ, I.F., BALOCCHI, O.A., KEMP, P.D., VALDES, C. 2009. Phenotypic variability in Holcus lanatus L. in southern Chile: a strategy that enhances plant survival and pasture stability. Crop \& Pasture Science 60: 768-777.

MONTALDO, P. 1973 (a). El Problema de la Clasificación de las Praderas Permanentes de Lluvia. Agro Sur 1: 3-10.

MIJANGOS, I., ALBIZU, I., EPELDE, L., AMEZAGA, I., MANDARTE, S., GARBISU, C. 2010. Effects of liming on soil properties and plant performance of temperate mountainous grasslands. Journal of Environmental Management 91: 2066-2074.

PACHECO, A. 1997. Dinámica vegetacional y rendimiento de una pradera establecida en base a especies nativas y naturalizadas en el Dominio Húmedo de Chile. Tesis Lic. Agr. Universidad Austral de Chile. 87 p.

PINOCHET, D. 1990. Fertilización de praderas permanentes en la zona sur. In: Latrille, L. (Ed.), Avances en Producción Animal. Universidad Austral de Chile, Facultad de
Ciencias Agrarias, Instituto de Producción Animal. Valdivia, pp. 181-209.

PINOCHET, D., BALOCCHI, O., NANNIG, P., FUENTES, R. 1999. Respuesta al nitrógeno de diferentes gramíneas forrajeras. Producción de Materia Seca. XXIV Reunión anual de la Sociedad Chilena de Producción Animal. Temuco, pp. 7-8.

PINOCHET, D., MENDOZA, J., GALVIS, A. 2000. Potencial de mineralización de nitrógeno de un Hapludand condistintos manejos agrícolas. Ciencia e Investigación Agraria 27: 97-106.

PINTO, M., RODRÍGUEZ, M., BESGA, C., BALCAZAR, N., LOPEZ, F.A. 1995. Effects of Linz-Donawitz (LD) slag on soil properties and pasture production in the Basque country (Northern Spain). New Zealand Journal of Agricultural Research 38: 143-155.

POOZESH, V., CASTILLON, P., CRUZ, P., BERTONI, G. 2010. Re-evaluation of the liming-fertilization interaction in grasslands on poor and acid soils. Grass and Forage Science 65: 260-272.

SAS INSTITUTE. 2006. SAS OnlineDoc 9.1.3. Cary, NC, USA: SAS Institute Inc.

SIEBALD, E., MATZNER, M., BECKER, F. 1983. Mejoramiento de praderas naturales del Llano Central de la X Región. Instituto de Investigaciones Agropecuarias (INIA). Estación Experimental Remehue. Osorno, Chile. Boletín Técnico № 51.20 p.

SIEBALD, E., NAVARRO, H., IRAIRA, S., SANTANA, R. 2002. Mejoramiento de praderas naturalizadas de la precordillera andina y de la costa de Llanquihue, Xa región. XXVII Reunión anual de la Sociedad Chilena de Producción Animal. Chillán, pp. 25-26.

TEUBER, N., ELIZALDE, H. 1999. Efecto del corte en diferentes estados fenológicos de la ballica perenne en la evolución con trébol blanco. Agro Sur 27: 94-104.

THARMARAJ, J., CHAPMAN, D.F., NIE, Z.N., LANE, A.P. 2008. Herbage accumulation, botanical composition, and nutritive value of five pasture types for dairy production in southern Australia. Australian Journal of Agricultural Research 59: 127-138.

TILMAN, D. y DOWNING, J. 1994. Biodiversity and stability in grasslands. Nature 367: 363-365.

TOW, P. 2001. Competition and succession in pastures. CABI, Oxon, UK.

WEDDERBURN, M., ADAM, K., GREAVES, L., CARTER, J. 1996. Effect of oversown ryegrass (Lolium perenne) and white clover (Trifolium repens) on the genetic structure of New Zealand hill pastures. New Zealand Journal of Agricultural Research 39: 41-52. 